\title{
Problems at the interface between heavy flavor physics, QCD and hadron spectroscopy
}

\author{
Harry J. Lipkin ${ }^{a, b, c 1}$ \\ a Department of Particle Physics Weizmann Institute of Science, Rehovot 76100, Israel \\ 'b School of Physics and Astronomy, Raymond and Beverly Sackler Faculty of Ezact \\ Sciences, Tel Aviv University, Tel Aviv, Israel \\ 'Eigh Energy Physics Division, Argonne National Laboratory, Argonne, IL 60439-4815, \\ USA
}

\begin{abstract}
.
- Pentaquark. Why it is important and how new technologies (vertex detectors) suggest drastically different approaches from the search used by Ashery et al.

- Problems in $B$ decays with implications for heavy quaik decays to excited light quark states like the al.

- Problems in $\mathrm{B}$ and $\mathrm{D}$ decays to final states including $\eta$ and $\eta^{\prime}$ indicating that standard quark mixing might not hold.

- Possible contributions of hybrid quarkonium states to B decays.

- Heavy fiavor decays to $\omega \pi$ which disagree with conventional expectations.

- Possible new spin effects in $\Lambda_{b}$ decay and the effect on the lifetime difference between the $A_{b}$ and $B$ mesons.
\end{abstract}

\section{Introduction - What is a Hadron? - The Blind Men and the Elephant}

Although QCD is supposed to explain everything, present attempts to describe hadrons remind us of the story of the blind men and the elephant.

1) Supported in part by grant No. [-0304-120-.07/93 from The German-Israeli Foundation for Scientific Research and Development and by the U.S. Department of Energy, Division of High Energy Physics, Contract W-31-109-ENG-38. 


\section{DISCLAIMER}

This report was prepared as an account of work sponsored by an agency of the United States Government. Neither the United States Government nor any agency thereof, nor any of their employees, make any warranty, express or implied, or assumes any legal liability or responsibility for the accuracy, completeness, or usefulness of any information, apparatus, product, or process disclosed, or represents that its use would not infringe privately owned rights. Reference herein to any specific commercial product, process, or service by trade name, trademark, manufacturer, or otherwise does not necessarily constitute or imply its endorsement, recommendation, or favoring by the United States Government or any agency thereof. The views and opinions of authors expressed herein do not necessarily state or reflect those of the United States Government or any agency thereof. 


\section{DISCLAIMER}

Portions of this document may be illegible in electronic image products. Images are produced from the best available original document. 
- A pion is a Goldstone Boson - A proton is a Skyrmion

- A pion is two-thirds of a proton. Data still fits simple prediction $\sigma_{\text {tot }}\left(\pi^{-} p\right) \approx(2 / 3) \cdot \sigma_{\text {tot }}(p p)[1,2]$ better than $7 \%$ up to $310 \mathrm{Gev} / \mathrm{c}[3]$.

- Mesons and Baryons are made of the same quarks. Remarkable agreement with experiment for static properties and low lying excitations when both are described as simple composites of asymptotically free quasiparticles having an effective mass with exactly the same value for predicting hadron masses, magnetic moments and hyperfine splittings $: 4,0 j$.

- Lattice QCD gives all answers. If answer is wrong, get bigger lattice [6].

- Lattice QCD on the $H$ dibaryon contains interesting physics ignored by lattice technologists [5-7]. They disagree on whether the $H$ is bound. Refuse to consider the physics until much bigger lattices are arailable [8].

- Light (uds) SU(3) symmetry and heavy quark symmetry (cbt) are good.

- Light (uds) $S U(3)$ symmetry is bad. All nontrivial hadron states violate SC (3). All light $\mathrm{V}, \mathrm{A}$ and $\mathrm{T}$ mesons have good isospin symmetry with flavor mixing in (u.d) space and no $s \bar{s}$ component; e.g. $\rho, \dot{w}, A_{2}, f_{2}$.

- The s-quark is a heavy quark. Flavor mixing in mass eigeustates predicted by $\mathrm{SU}(3)$ is not there. Most nontrivial strange hadron states satisfy (scb) heavy quark symmetry with no flavor mixing.; e.g. $\phi, \psi, \Upsilon$.

Because of lack of time we consider only two topics listed in the abstract.

\section{The pentaquark search - why and low}

The possible existence of exotic hadrons remains a principal question in hadron spectroscopy and the understanding of how QCD describes binding of quarks and gluons into hadrons [5]. We still have no experimental information about short-range $(q q)_{6}$ or $(\bar{q} q)_{8}$ interactions in color sextet or color octet states. All successes of the constituent quark model with a two-body color-exchange interaction $[9,10]$ and all hadron spectroscopy without exotics including scattering still probe only $(\bar{q} q)_{1}$ and the $(q q)_{3 \times}$ interactions in color singlet and color antitriplet states. Hadron-hadron scattering in the $(u, d, s)$ sector is dominated either by $q q$ repulsion between two quarks of same $\mathrm{Ha}$ vor or by resonances produced by annihilation of $\bar{q} q$ pair of the same flavor. Short-range $(q q)_{6}$ or $(\vec{q} q)_{8}$ interactions are observable only with more than three flavors in realistic scattering experiments with no common flavor between beam and target. The first question to test is whether these short range interactions can produce an exotic multiquark bound state.

Two prime candidates for possibly bound exotics are $H$ dibaryon [11] and pentaquark .12-14]. Original multiquark calculations indicate equal gains in 
hyperfine energy respectively over $\Lambda \Lambda$ and $D, p$ threholds by recoupling color and spins. A lattice gauge calculation [6] showed binding of $H$ prevented by repulsive $\Lambda-\Lambda$ interaction generated by quark exchange $[5,8]$. No such exchange is possible for $D_{s} p$. Thus the pentaquark seems better candidate [5]. Comparing results for these cases may provide considerable insight into understanding QCD physics in multiquark systems even if the pentaquark is not bound. Both experimental searches and lattice gauge calculations for the pentaquark are of interest. But no such lattice calculation is planned.

In experimental searches every event with a proton from a secondary vertex indicates a weakly decaying baryon. It cannot be a statistical fluctuation of known physics. Plans for future experiments and data analyses from ongoing experiments should recognize this point [5] which has not been previously noted $[15,16]$. Pentaquark candidate events seen in the recent Fermilab experiment [17] are suggestive but not yet conclusive, because of experimental uncertainties. Subsequent experiments with better vertex detectors that cleanly distinguish between particles from primary and secondary vertices and gcod particle $I D$ which unambiguously identify a proton will not need striking decay signatures like $p \phi \pi^{-}$and should be considered as open searches for new weakly decaying baryons rather than only the pentaquark. They might even find new physics beyond the standard model.

\section{Effects of hyperfine splitting on $\Lambda_{b}$ Decay}

Hadron specroscopy effects have been shown to contribute to the so far unexplained enhancement of the $\Lambda_{b}$ lifetime relative to the $B$ lifetime [18]. Consider a model; e.g. factorization, where the hadronic decay is descrihed at the quark level as $b \rightarrow c+X, X$ denotes any hadron or multihadron state, and the charmed quark combines with the spectator antiquark in the $B$ decay and with the spectator diquark in the $\lambda_{b}$ decay. If the $(S=0, I=0)$ spin and isospin of the spectator diquark are not changed during the transition, the $\Lambda_{b} \rightarrow \Sigma_{c}+X$ and $\Lambda_{b} \rightarrow \Sigma_{c}^{-}+X$ decays are forbidden while the $\Lambda_{b} \rightarrow \Lambda_{c}+X$ is allowed. This "spectator spin-isospin conservation" leads to a number of interesting predictions which can be checked by future experiments. The $\Lambda_{b}$ will decay to a $\Lambda_{c}$ and not a $\Sigma_{c}$ or $\Sigma_{c}{ }^{*}$ if the spin-zero diquark picks up a charmed quark, to a $\Lambda$ and not a $\Sigma$ or $\Sigma^{*}$ if it picks up a strange quark, and to a nucleon and not a $\Delta$ if it picks up a nonstrange quark.

This spin-isospin effect enhances baryons decays. This is simply seen in a quark-hadron duality approach where the $B$ and $\Lambda_{b}$ have the same phase space if spin effects are neglected and decay phase space is given by a sum over all final states without regard to spin form factors. Introducing spectator spin-isospin conservation does not affect the $B$ decay but enhances the $\Lambda_{b}$ decay by always choosing the lower $(S=0, I=0)$ spectator state and increasing the available phase space. 
A quantitative estimate with a toy model using experimental masses shows the following results for the ratios of semileptonic partial widths and lifetimes,

$$
\frac{\Gamma\left(\Lambda_{b}\right)}{\Gamma(B)}=1.07 ; \quad \frac{\tau\left(\Lambda_{b}\right)}{\tau(B)}=0.938
$$

These results suggest (1) that phase space effects must be carefully taken into account using exclusive final states in lifetime calculations which compare the $B$ and $\Lambda_{b}$ decays; (2) that the validity of the spectator spin conservation model should be tested with new data and new analyzes.

\section{Acknowledgement}

Many stimulating discussions with the Tel Aviv experimental group $[17,16]$ are gratefully acknowledged and in particular Danny Ashery, Sharon May-Tal Beck, Gilad Hurvits, Jechiel Lichtenstadt and Murray Moinester.

\section{REFERENCES}

1. E. M. Levin and L. L. Frankfurt, Zh. Eksperim. i. Theor. Fiz.-Pis'ma Redakt (1965) 105; JETP Letters (1965) 65

2. H.J. Lipkin and F. Scheck, Phys. Rev. Lett. 16 (1966) 71

3. Harry J. Lipkin, Physics Letters B335 (1994) 500

4. Ya. B. Zeldovich and A.D. Sakharov, Yad. Fiz 4 (1966)395; Sov. J. Nucl. Phys. 4 (1967) 283

5. Harry J. Lipkin, Nucl. Phys. A, in press and references therein

6 P. MacKenzie and H. Thacker, Phys. Rev. Letters 65, 2539 (1985)

7. Murray A. Moinester, Carl B. Dover and Harry J. Lipkin, Phys. Rev. C46 (1992) 1082

8. H. Thacker, private communication

9. H.J. Lipkin, Phys. Lett. 45B (1973) 267

10. A. De Rujula, H. Georgi and S.L. Glashow, Phys. Rev. D12 (1975) 147

11. R. L. Jaffe, Phys. Rev. Lett. 38, (1977) 195

12. Harry J. Lipkin, in Hadrons, Quarks and Gluons, Proceedings of the Hadronic Session of the XXIInd Rencontre de Moriond, Edited by J. Tran Thanh Van, Editions Frontieres, Gif Sur Yvette - France (1987), p.691

13. Harry J. Lipkin, Phys. Lett. 195B, (1987) 484

14. C. Gignoux, B. Silvestre-Brac and J. M. Richard, Phys. Lett. B193 (1987) 323

15. Harry J. Lipkin, Nucl. Phys. B(Proc. Suppl.) 21 (1991) 258

16. M. A. Moinester, D. Ashery, L. G. Landsberg and H. J. Lipkin, Zeitschrift fur Physik A 356 (1996) 207

17. Daniel Ashery, these proceedings

18. Harry J. Lipkin and Patrick J. O'Donnell, Physics Letters B409 (1997) 412 